\title{
Respective interest of T2 mapping and diffusion tensor imaging in assessing porcine knee cartilage with MR at 3 Teslas
}

\author{
Bailiang Chen ${ }^{\mathrm{a}, \mathrm{b}, *}$, Emilie Roeder ${ }^{\mathrm{c}, *}$, Pierre-André Vuissoz ${ }^{\mathrm{a}, \mathrm{b}}$, Pierre Gillet $^{\mathrm{c}, * *}$, \\ Jacques Felblinger ${ }^{\mathrm{a}, \mathrm{e}}$, Marine Beaumont ${ }^{\mathrm{a}, \mathrm{d}, * * *}$ and Astrid Pinzano ${ }^{\mathrm{b}, * * *}$ \\ ${ }^{a}$ IADI, Université de Lorraine, Nancy, France \\ ${ }^{\mathrm{b}}$ U947, INSERM, Nancy, France \\ ${ }^{\mathrm{c}}$ UMR 7365/CNRS, Université de Lorraine, IMOPA, Nancy, France \\ ${ }^{\mathrm{d}}$ CIC-IT 801, INSERM, Nancy, France \\ ${ }^{\mathrm{e}}$ Pôle Imagerie, CHU Nancy, Nancy, France
}

\begin{abstract}
Non-invasive quantitative assessment of articular cartilage integrity is essential for early detection and evaluation of osteoarthritis (OA) and for the follow-up of stem-cell-driven cartilage engineering. In this study, we investigated the feasibility of exploiting diffusion tensor imaging (DTI) on porcine knee joints with a clinical magnetic resonance (MR) scanner to extract micro-structural information in order to complement biochemical information quantified by T2 maps. We propose an MR protocol for quantifying T2 and cartilage microstructure with diffusion MR on a clinical scanner. Preliminary results were obtained on four pig knee joints using a 3 T GE clinical MRI scanner and an 8-channel knee coil array. The measured cartilage volume, T2 values, apparent diffusion coefficient and fractional anisotropy (FA) of femoral and tibial cartilage were respectively $9.8 / 2.3 \mathrm{~mm}^{2}, 67.0 / 56.1 \mathrm{~ms}, 1.3 / 1.3 \times 10^{-3} \mathrm{~mm}^{2} / \mathrm{s}$ and $0.4 / 0.3$. This new protocol has the potential to be combined in vivo with quantitative assessment of both cartilage degradation and restoration in osteoarthritis.
\end{abstract}

Keywords: Osteoarthritis, stem cell therapy, quantitative assessment, MR, diffusion tensor imaging

\section{Introduction}

Assessment of both the integrity of articular cartilage and cartilage repair using non-invasive techniques is a key element in the diagnosis and treatment of osteoarthritis (OA) including stem cell therapies [1,2]. Proteoglycan and collagen are responsible for the mechanical properties of cartilage. In the initial stages of OA, loss of proteoglycan and disruption of the collagen network occur, extending degradation of the hyaline cartilage degradation. In clinical practice, magnetic resonance imaging (MRI) is the current gold standard method to explore damage to articular cartilage [3]. Conventional MR methods are mostly restricted to either qualitative comparison by radiologists or morphological measurements,

\footnotetext{
*First co-author.

**Address for correspondence: Pierre Gillet, UMR 7365 CNRS-Université de Lorraine, "Ingénierie Moléculaire et Physiopathologie Articulaire", Biopôle de l'Université de Lorraine, Campus biologie-santé, Faculté de Médecine, Avenue de la forêt de Haye, BP 184, 54505 Vandoeuvre-lès-Nancy, France. E-mail: pierre.gillet@ univ-lorraine.fr.

${ }^{* * *}$ Equal seniorship.
} 
such as the volume and thickness of the cartilage $[3,4]$. However, such global morphological data fail to provide the biomechanical and physiologic information which is indispensable for early detection of $\mathrm{OA}$ and for monitoring cartilage repair [5].

To overcome the above clinical measurement problems, various sequences have been proposed and investigated to assess cartilage composition, among which delayed gadolinium-enhanced MRI for T1 mapping, T1 rho (spin-lock) imaging, T2 mapping and diffusion MR are considered to be the most promising techniques. T1 mapping with delayed gadolinium-enhanced MRI (dGEMRIC) allows quantitative measurement of glycosaminoglycan (GAG) content, as Gd-DTPA2-molecules enter the cartilage and concentrate in places where GAG content is low [4]. T1 $\rho$ (T1 rho) imaging monitors the interaction between motion-restricted molecules of water and their local environment. T1 $\rho$ values increase in damaged cartilage and are sensitive to changes in extracellular matrix, such as depletion of proteoglycan and the orientation and concentration of collagen fibre [6].

T2 mapping provides information on the interaction between water molecules, water molecules and the surrounding macromolecules and hence indirect information on the concentration and orientation of the collagen network [2,7]. T2 is very sensitive to changes in cartilage integrity. Damaged cartilage tissues have higher T2 values than normal cartilage. Nishioka et al. [6] reported a significant correlation between an increase in $\mathrm{T} 2$ values and the degree of degeneration of human cartilage, in particular the depletion of GAG observed by histological grading. Another encouraging alternative way to evaluate the composition of articular cartilage is diffusion MR, which exploits the molecular mobility of water protons. In healthy cartilage, extra-cellular components (e.g. the collagen network and proteoglycans) form a thick matrix, which limits the mobility of water molecules within the cartilage. This restriction causes a low apparent diffusion coefficient (ADC) at long diffusion time, whereas in a damaged cartilage matrix, the ADC is increased by the enhanced mobility of water. Diffusion tensor imaging (DTI) also has the advantage that variations in ADC values in cartilage or other tissues can be mapped [7].

Several studies focused on water diffusion in articular cartilage. Burstein et al. [8] showed that selfdiffusivity of water in cartilage increased when enzymatic degradation of cartilage was induced using trypsin. Xia et al. [9] confirmed Burstein's observation by demonstrating that enzymatic or chemical degradation of canine cartilage enhanced ADC values. However, Xia's group found no evidence of a correlation between variation in ADC and GAG depletion. Mlynárik et al. [10] correlated changes in ADC with a decrease in proteglycan content in human arthritic cartilage, and compared with T1 values measured by Gd-DTPA enhanced T1 mapping and safranin-O in histological section.

More recently, Welsch et al. [11] demonstrated that T2 mapping and diffusion weighted imaging provide complementary information on the monitoring of cartilage repair after various surgical treatments. These authors showed that T2 mapping could be used to differentiate two neo-synthesized repair tissues, while diffusion MR could differentiate between healthy cartilage and repair tissue. These results of diffusion-weighted imaging were obtained in a recent study by Apprich et al. [12]. However, all these studies only investigated water diffusion in a single direction within the cartilage without considering the specific directional feature of water diffusion within cartilage caused by the orientation of the collagen fibers. Thus, such measurements of diffusion direction may be biased.

The other feature which reflects cartilage quality is its microstructure, i.e. the organization of the collagen fibers, which determines the mechanical competence of the cartilage. DTI can be used to understand its microstructure. Cartilage is composed of at least six diffusion weighted acquisitions (as used in [8-12]) in different directions (usually uniformly distributed on the surface of the sphere), in order to solve the aforementioned directional dependency problem by reconstructing a diffusion tensor. Using the DTI technique on bovine and human articular cartilage, Filidoro et al. [13] demonstrated that 
the orientation of the maximum eigenvectors appears to express the arrangement of collagen fibres in arcades. Raya et al. [14] suggested that ADC are dependent on both proteoglycan and collagen contents and argued that ADC could help distinguish proteoglycan depletion from damage to collagen fibres. They also suggested that the fraction anisotropy (FA) and the first eigenvector were specific to the collagen architecture of cartilage. Meder et al. [15] used the DTI technique to investigate the collagen fiber microstructure of articular cartilage by analysing the main orientation of diffusion tensors.

A combination of several MR contrast mechanisms may be a more sensitive approach to assess the internal structural of cartilage integrity and hence of damage to the cartilage at an earlier stage, or to quantitatively assess the efficacy of stem cell therapy. However, most of these observations were made on high magnetic field small animal scanners. To transfer such monitoring techniques to a clinical scenario (i.e. on a clinical scanner with a much lower magnetic field) is an important step in promoting stem cell therapy and the aforementioned sequences for clinical applications. The aim of our work was to investigate the feasibility of applying two MRI techniques, T2 mapping and DTI to characterise articular cartilage microstructure in pig knee joints on a 3 T MR scanner.

\section{Materials and methods}

\subsection{Sample preparation}

Four samples of 18 month-old porcine knee joints were obtained fresh from a local abattoir. Samples were maintained frozen at $-20^{\circ} \mathrm{C}$ prior to analysis. Twenty-four hours before acquisition, the samples were defrosted at $4{ }^{\circ} \mathrm{C}$ then at room temperature for $1 \mathrm{~h}$. Two knee joints were embedded in a $4 \%$ agarose (Sigma, France) gel to minimize geometric distortion during acquisition (preparation 1). Two other samples were imaged without being embedded in gel (preparation 2).

\subsection{Magnetic resonance imaging}

MRI experiments were undertaken at room temperature on a $3 \mathrm{~T}$ clinical MR scanner (Signa HDx, General Electric Healthcare, WI, USA). The proposed protocol is composed of three sequences: a 3D CUBE (a 3D fast spin echo sequence with variable flip angles and parallel imaging in two dimensions) [16] for cartilage morphology; a multi-echo (8 echoes) spin echo sequence for T2 maps [17]; and a double-echo pulsed gradient spin echo sequence with echo planar imaging (EPI) readout for DTI acquisition with a $b$-value equal to $400 \mathrm{~s} / \mathrm{mm}^{2}$. Six diffusion gradients were applied with one non-diffusion weighted acquisition ( $b$-value equals 0 ). The in-plane resolution and slice thickness were set to maximum with a desired SNR using 16 NEX. To reduce geometrical distortion, parallel imaging option with acceleration factor 2 and partial Fourier option were also applied. The detailed imaging parameters are listed in Table 1.

The cartilage morphology was analysed based on the 3D CUBE acquisition, and 3D anatomical models were reconstructed to understand its surface geometry. The sample's volume was measured using MITK open-source software [18]. The T2 map of each sample was created to calculate the global femur and tibia cartilage T2 values was programmed in MATLAB (R2007b, Natick, MA) using in-house software. The diffusion tensors were reconstructed using a standard linear regression algorithm with least squares. The apparent mean diffusivity and fractional anisotropy (FA) were calculated voxel-wise. The apparent mean diffusivity was calculated for both tibial and femoral cartilage of all four samples. The main orientation of the diffusion tensors was derived via eigensystem decomposition. The analyses were 
Table 1

Imaging protocol

\begin{tabular}{lccccccc}
\hline Sequence name & $\begin{array}{c}\text { Time duration } \\
(\mathrm{min})\end{array}$ & $\begin{array}{c}\text { FoV } \\
(\mathrm{cm})\end{array}$ & $\begin{array}{c}\text { Acquisition } \\
\text { matrix }\end{array}$ & $\begin{array}{c}\text { TR/TE } \\
(\mathrm{ms})\end{array}$ & $\begin{array}{c}\text { Slice } \\
\text { thickness }\end{array}$ & $\begin{array}{c}\text { NEX } \\
\text { Acquisition } \\
\text { plane }\end{array}$ \\
\hline SPGR T1 3D & 7.5 & 14 & $512 \times 224 \times 74$ & $25.9 / 9.8$ & 1 & 1 & $\begin{array}{c}\text { Sagittal } \\
\text { CUBE 3D }\end{array}$ \\
CU.3 & 14 & $416 \times 384 \times 128$ & & 0.6 & 0.5 & $\begin{array}{c}\text { Sagittal } \\
\text { Axial }\end{array}$ \\
Dual echo PGSE & 18.5 & 14 & $128 \times 128(\times 32)$ & $9900 / 81.1$ & 2.4 & 16 & \\
$\quad$ (DTI) with EPI & & & & & & & \\
$\quad$ read-out & 10 & 14 & $384 \times 384 \times 20$ & $1500 /(7.6,15.3$, & 4 & 0.5 & Sagittal \\
T2 mapping & & & & $22.9,30.5,38.2$, & & & \\
& & & & $45.8,53.4,61.1)$ & & & \\
\hline
\end{tabular}

carried out using open source software CAMINO [19] and in-house software with MATLAB (R2007b, The MathWorks, Natick, MA, USA). The voxel-wise 3D diffusion tensor plot was created using a third party MATLAB software [20].

\section{Results}

Between the two methods of sample preparation, preparation 1 introduced much less geometrical distortion than preparation 2 , as expected in diffusion sequences. There was no notable distortion due to the susceptibility effects for all other sequences included in the protocol using either method of preparation. An example of a reconstructed femoral and tibial cartilage model (from the CUBE dataset of pig knee sample 1) is shown in Fig. 1. In most places, the femoral cartilage appears to be thicker than the tibial cartilage, with a volume of $9.2 \mathrm{~mm}^{3}$ than $2.0 \mathrm{~mm}^{3}$ for the tibial cartilage. Sampled diffusion weighted images (in the axial plane) are presented in Fig. 2. When compared to the corresponding non-diffusion weighted images (Fig. 2(a)), the intensity in the cartilage region (the femoral cartilage is indicated by yellow arrows) decreased by $35.6 \%$ after the diffusion gradients were applied (Fig. 2(b)).

Diffusion parameters are presented as a trace map and a FA map and examples (from sample 4) in Fig. 3. The cartilage region corresponds to the hyper intensity in the trace map and low intensity in the FA map. The T2 values of the cartilage varied between 50 and $65 \mathrm{~ms}$ (one slice of the T2 map of sample 1 is shown in Fig. 4). The internal cartilage structure may be indicated by the principal orientation of diffusion tensors (indicated by the first eigenvectors), as demonstrated in Fig. 5. A 2D axial plane colourcoded FA map is shown in Fig. 5(a) including a zoom to show the cartilage more clearly (Fig. 5(b), the cartilage area is delineated by yellow lines). Red, green and blue colours correspond to the $x, y$ and $z$ imaging axes respectively. The projection of the principal eigenvectors in each voxel are overlaid on top of the colour-coded map and scaled with the corresponding eigenvalues. A 3D tensor plot in the same region is also presented in Fig. 5(c).

According to the figure, the diffusion tensors inside cartilage region mainly orientate along the $z$ axis. This may indicate that the collagen fibres in this part are organized along the $z$ axis. This part corresponds to the lower part of the femur condyle, whose surface winds through the axial plane. The volume and MR properties of the femoral and tibial cartilage of each sample are listed in Table 2. The averaged cartilage volume for a healthy piglet is $9.8( \pm 0.5) \mathrm{mm}^{3}$ for femoral cartilage and $2.3( \pm 0.3) \mathrm{mm}^{3}$ for tibial cartilage. The averaged global T2 values for femoral and tibial cartilage are 67.0 and $56.1 \mathrm{~ms}$, respectively. The global averaged ADC value (computed only from samples immersed in gel) was $1.3 \times$ $10^{-3} \mathrm{~mm}^{2} / \mathrm{s}$ for both femoral and tibial cartilage. 


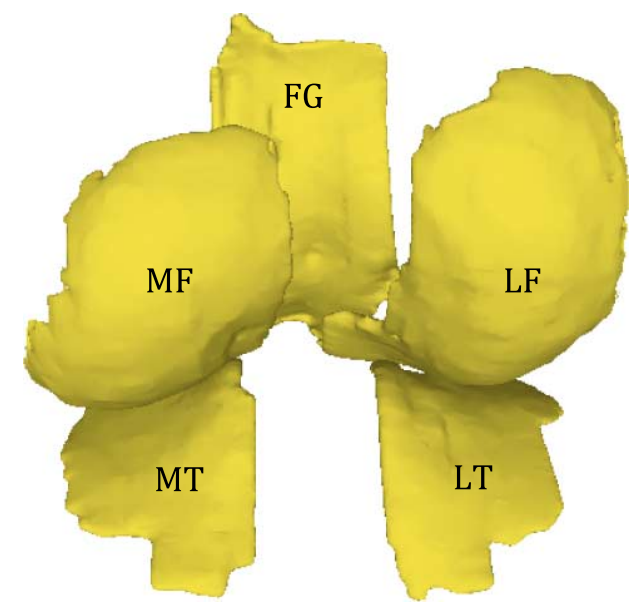

Fig. 1. 3D morphological model of femoral and tibial cartilage (posterior view). M: medial, L: lateral, T: tibia, F: femur, FG: femoral groove. (Colors are visible in the online version of the article; http://dx.doi.org/10.3233/BME-130750.)

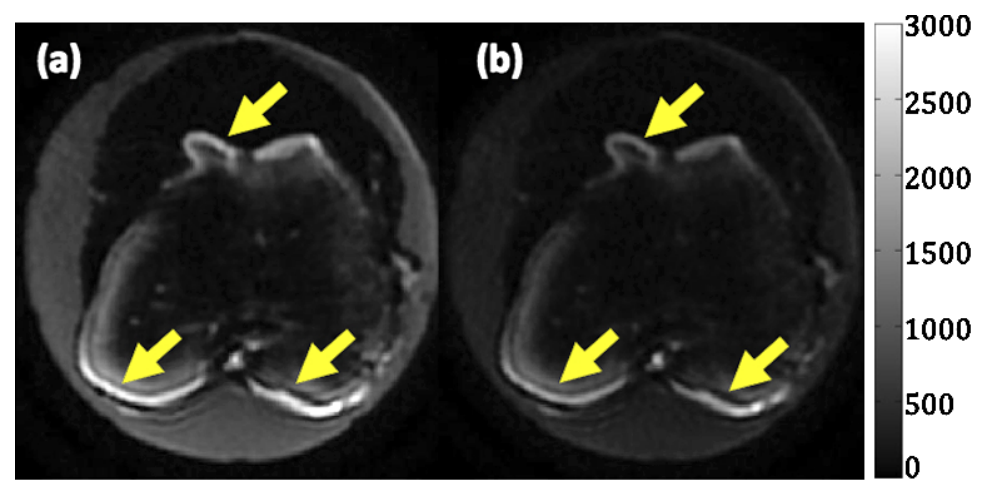

Fig. 2. Examples of acquired diffusion weighted data: (a) non-diffusion weighted image; (b) diffusion weighted image. Yellow arrows indicate cartilage regions. (The colors are visible in the online version of the article; http://dx.doi.org/10.3233/ BME-130750.)

\section{Discussion}

A clinical MR imaging protocol is proposed for the quantitative evaluation and monitoring of cartilage engineering, e.g. for osteoarthritis. We demonstrated the feasibility of this protocol on four piglet healthy knee joints. The current protocol is able to quantitatively characterise the 3D morphology, T2 and mean diffusion properties. The application included a diffusion tensor sequence and may also provide an opportunity to characterise the collagen fibre network. The total time required for the whole protocol is less than an hour (the maximum time allowed for examination of a patient) for future clinical translation. Since the T2 values are related to the water content of the cartilage matrix [2], while anisotropic diffusion tensor principal orientations are caused by anisotropic diffusion of water molecules related to the orientation of the collagen fibre [14], the two types of parametric maps can provide complementary insights into the cartilage.

The T2 maps of articular cartilage are a function of the water content of the tissue. In the present observation, the global T2 values of the femoral and tibial cartilage were 67.0 and $55.1 \mathrm{~ms}$ respectively, comparable to the values reported by Shiomi et al. in [21] in non-loading conditions. 


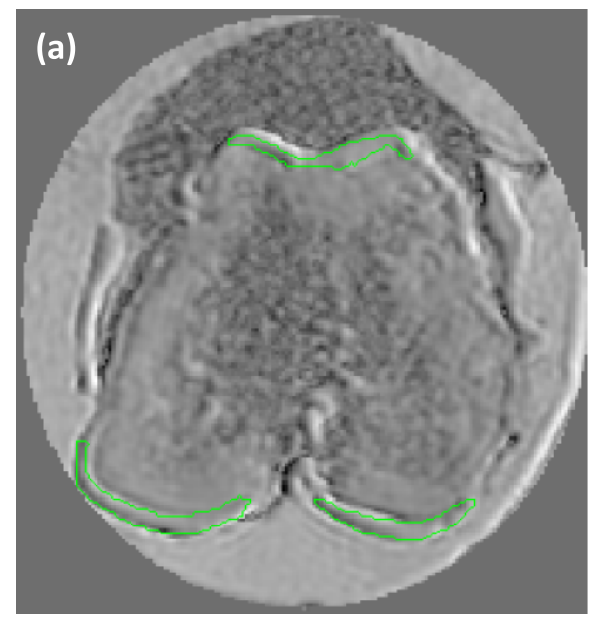
$\times{ }^{10-8}\left(\mathrm{~m}^{2} / \mathrm{s}\right)$

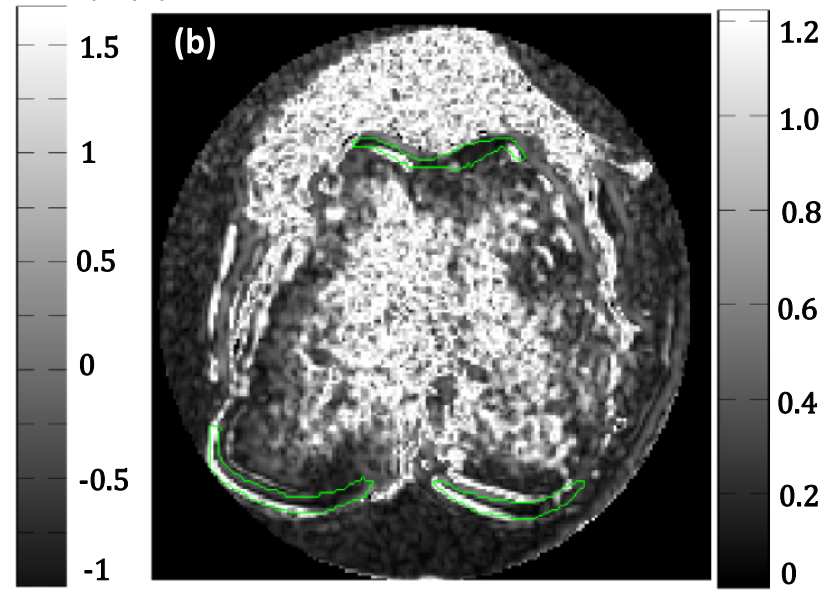

Fig. 3. Sampled trace map (a) and FA map (b) of the femoral cartilage region (delineated by green lines). (The colors are visible in the online version of the article; http://dx.doi.org/10.3233/BME-130750.)
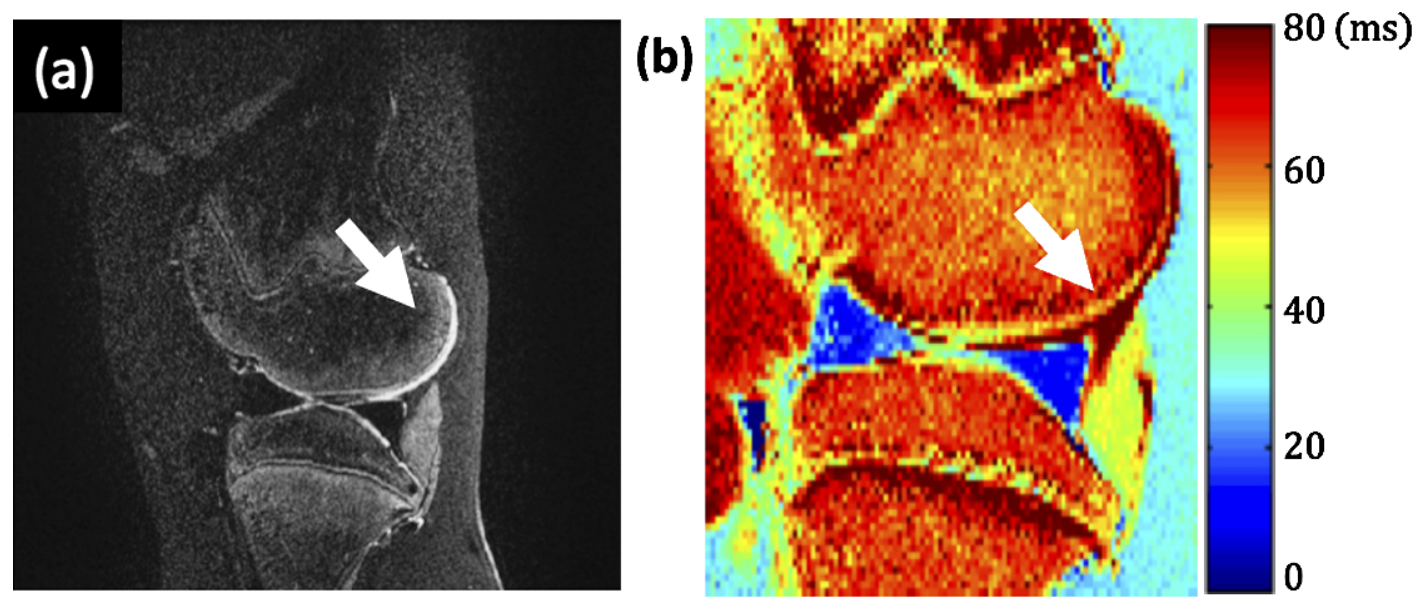

Fig. 4. Example of T2 maps (a) one slice of CUBE data; (b) the T2 map in the corresponding region (indicated by white arrows). (Colors are visible in the online version of the article; http://dx.doi.org/10.3233/BME-130750.)

On clinical MR scanners, diffusion protocols are seriously affected by various artefacts, among which geometrical distortion and susceptibility effects are the most serious. This is due to the echo planar imaging (EPI) readout. The strategy exploited here to reduce geometrical distortion uses the partial Fourier option and a parallel imaging setting and consequently reduces the total length of the $k$-space trajectory.

The susceptibility effects in this ex vivo experiment mainly occurred in the interface between the knee joint and the air. This problem is not serious in sequences such as gradient or spin echoes, but is enhanced by a EPI sequence. We consequently investigated two ways of preparing the samples: one in ambient air, the other immersed in gel. As expected, the preparation using gel to immerse the sample led to less signal loss and geometrical distortion caused by susceptibility. However, some signal void regions were still observed. These were caused by air bubbles generated while the sample were being immersed in the 


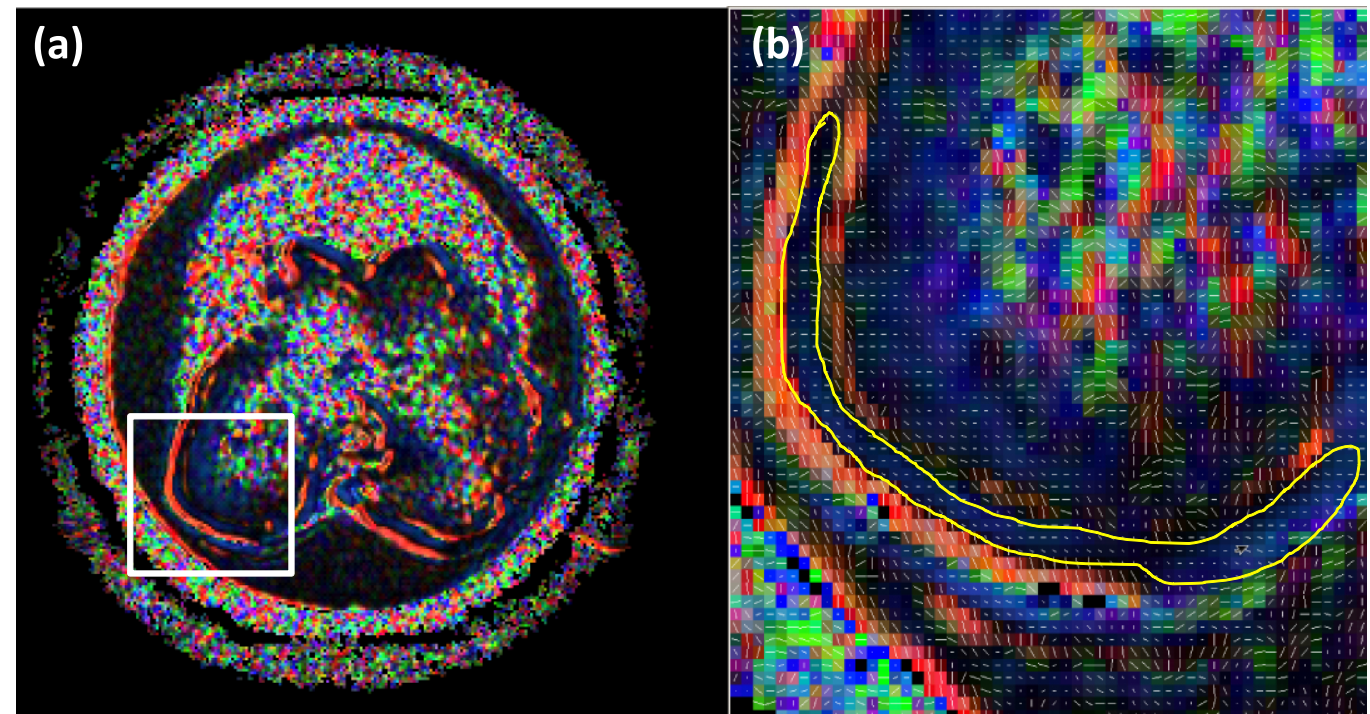

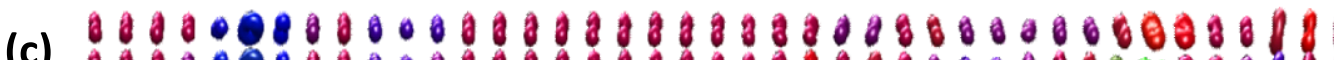

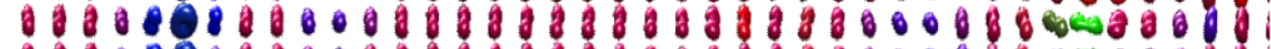

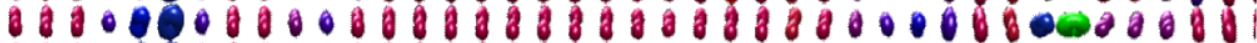

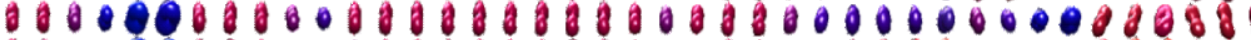

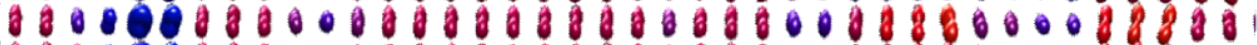

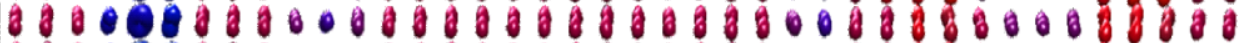

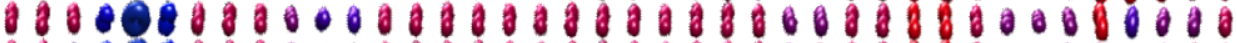

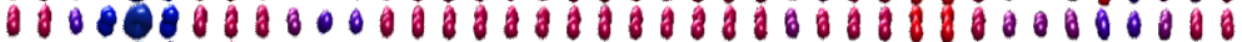

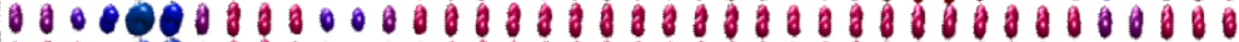

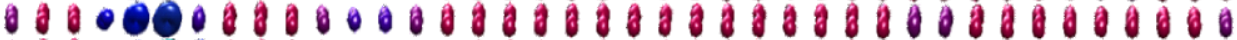

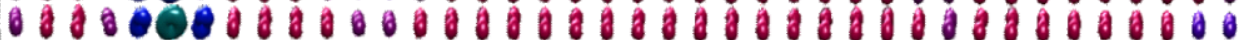

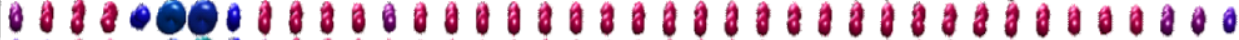

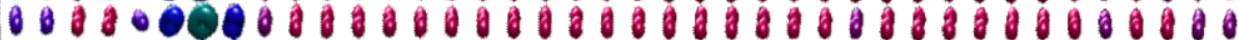

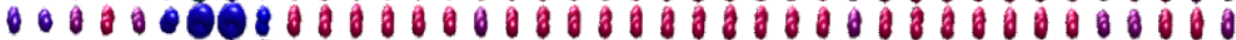

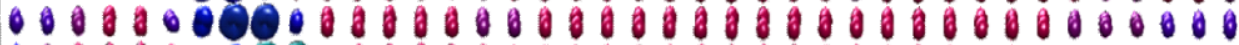

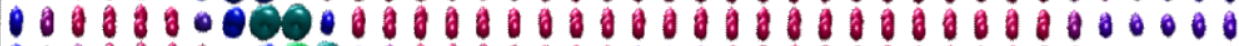

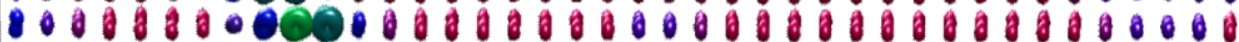
O000月a

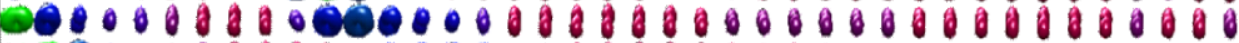

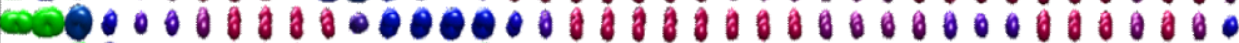

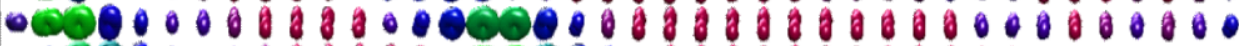

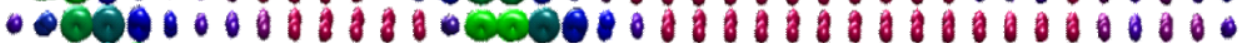

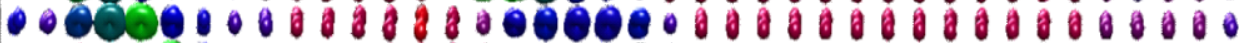

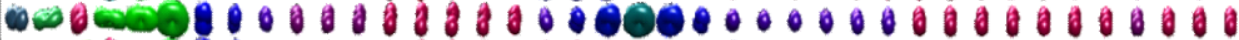

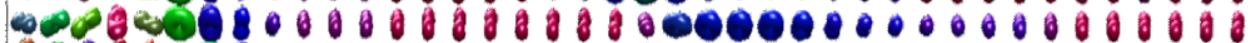

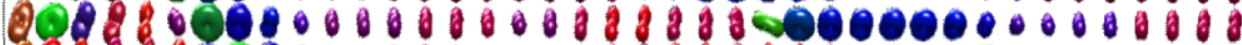

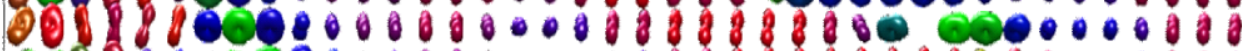

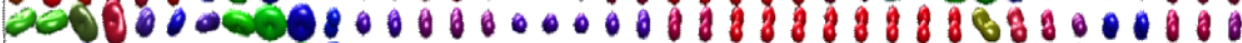
- 10000000000000000 月

Fig. 5. Main orientations of diffusion tensors in the cartilage region: (a) colour-coded principal eigenvectors weighted by FA values. Boxed region in white in (a) is enlarged in (b) to show 2D projection of eigenvectors scaled with corresponding eigenvalues are plotted in each voxel. 3D plot of diffusion tensors in the same region is presented in (c). (Red: $x$ axis, green: $y$ axis, blue: $z$ axis.) (The colors are visible in the online version of the article; http://dx.doi.org/10.3233/BME-130750.) 
Table 2

Measured sample parameters

\begin{tabular}{llcccc}
\hline Sample No. & Volume & $\begin{array}{c}\text { Global T2 value } \\
\left(\mathrm{mm}^{3}\right)\end{array}$ & $\begin{array}{c}\text { Global ADC } \\
\left(\times 10^{-3} \mathrm{~mm}^{2} / \mathrm{s}\right)\end{array}$ & $\begin{array}{c}\text { Fractional anisotropy } \\
(\text { FA })\end{array}$ \\
\hline 1 & Femoral cartilage & 9.2 & 69.1 & - & - \\
& Tibial cartilage & 2.0 & 52.7 & - & - \\
2 & Femoral cartilage & 10.3 & 68.5 & - & - \\
& Tibial cartilage & 2.0 & 55.6 & - & 0.3 \\
3 & Femoral cartilage & 9.5 & 68.0 & 1.5 & 0.3 \\
& Tibial cartilage & 2.4 & 59.8 & 1.3 & 0.5 \\
\multirow{4}{*}{ Overall } & Femoral cartilage & 10.0 & 63.0 & 1.0 & 0.4 \\
& Tibial cartilage & 2.7 & 57.5 & 1.3 & 0.4 \\
\hline
\end{tabular}

gel. These regions were excluded when the $\mathrm{T} 2$ and $\mathrm{ADC}$ values were being calculated but were included for quantification of cartilage volume.

Techniques such as dGEMRIC and T1 rho mapping are also considered to be beneficial for early detection of OA and for evaluation of cartilage engineering. The drawback of dGEMRIC imaging techniques is that they take a long time, not only for the acquisition itself, which may involve patient's motion artefacts, but also because of the long waiting time from injection of the contrast agent to the beginning of acquisition (i.e. $90 \mathrm{~min}$ ) [5]. Although results of $\mathrm{T} 1$ rho mapping are promising, the limited accessibility of this sequence on commercial MR systems is responsible for its non-use in clinical applications. At the present time, we are focusing on establishing a clinical MR protocol which respects the time allowed for patient examination (normally $1 \mathrm{~h}$ ). Consequently, only T2 mapping and DTI can satisfy this requirement.

\subsection{Limitations of the study}

Although the current protocol is promising in providing satisfactory images and enabling quantitative evaluation of cartilage morphology, composition and micro-structure, more experiments with both healthy and treated/diseased knee joint samples are needed to further demonstrate the efficacy of the protocol's ability to quantitatively characterise cartilage restoration/defection. For clinical diffusion protocols, the axial plane is preferred, while standard cartilage T2 maps are mainly acquired in the sagittal plane. Histological correlations will advance our understanding of the respective relationships between these two parametric maps, as biomarkers for the assessment of cartilage engineering.

\section{Conclusion}

The proposed protocol is applicable for quantification of pig femoral and tibial cartilage morphology, composition and internal structure ex vivo on a clinical MR scanner within the clinically allowed time. It has the potential to be used in combination for the quantitative assessment of healthy, diseased and engineered cartilage in vivo. 


\section{Acknowledgements}

This work was supported by grants from Agence Nationale de la Recherche - Programme ANR Blanc 2009: Cartilage Engineering and MRI: Assessment of Biointegration and Biofunctionality in the Rat (CEMABIR, ANR-09-BLANC-0150-01) and grants from the Région Lorraine Communauté Urbaine du Grand Nancy and Conseil Général de Meurthe et Moselle.

\section{References}

[1] S.E. Domayer, G.H. Welsch, R. Dorotka, T.C. Mamisch, S. Marlovits, P. Szomolanyi and S. Trattnig, MRI monitoring of cartilage repair in the knee: a review, Semin. Musculoskelet. Radiol. 12(4) (2008), 302-317.

[2] S. Trattnig, S. Domayer, G.W. Welsch, T. Mosher and F. Eckstein, MR imaging of cartilage and its repair in the knee a review, Eur. Radiol. 19(7) (2009), 1582-1594.

[3] R. Kijowski and G.E. Gold, Routine 3D magnetic resonance imaging of joints, J. Magn. Reson. Imaging 33(4) (2011), 758-771.

[4] G.E. Gold, C.A. Chen, S. Koo, B.A. Hargreaves and N.K. Bangerter, Recent advances in MRI of articular cartilage, AJR Am. J. Roentgenol. 193(3) (2009), 628-638.

[5] Y.X. Wang, J.F. Griffith and A.T. Ahuja, Non-invasive MRI assessment of the articular cartilage in clinical studies and experimental settings, World J. Radiol. 2(1) (2010), 44-54.

[6] H. Nishioka, J. Hirose, E. Nakamura, Y. Oniki, K. Takada, Y. Yamashita and H. Mizuta, T1 $\rho$ and T2 mapping reveal the in vivo extracellular matrix of articular cartilage, J. Magn. Reson. Imaging 35(1) (2012), 147-155.

[7] M.D. Crema, F.W. Roemer, M.D. Marra, D. Burstein, G.E. Gold, F. Eckstein, T. Baum, T.J. Mosher, J.A. Carrino and A. Guermazi, Articular cartilage in the knee: current MR imaging techniques and applications in clinical practice and research, Radiographics 31(1) (2011), 37-61.

[8] D. Burstein, M.L. Gray, A.L. Hartman, R. Gipe and B.D. Foy, Diffusion of small solutes in cartilage as measured by nuclear magnetic resonance (NMR) spectroscopy and imaging, J. Orthop. Res. 11(4) (1993), 465-478.

[9] Y. Xia, T. Farquhar, N. Burton-Wurster, M. Vernier-Singer, G. Lust and L.W. Jelinski, Self-diffusion monitors degraded cartilage, Arch. Biochem. Biophys. 323(2) (1995), 323-328.

[10] V. Mlynárik, I. Sulzbacher, M. Bittsanský, R. Fuiko and S. Trattnig, Investigation of apparent diffusion constant as an indicator of early degenerative disease in articular cartilage. J. Magn. Reson. Imaging 17(4) (2003), 440-444.

[11] G.H. Welsch, S. Trattnig, S. Domayer, S. Marlovits, L.M. White and T.C. Mamisch, Multimodal approach in the use of clinical scoring, morphological MRI and biochemical T2-mapping and diffusion-weighted imaging in their ability to assess differences between cartilage repair tissue after microfracture therapy and matrix-associated autologous chondrocyte transplantation: a pilot study, Osteoarthritis Cartilage 17(9) (2009), 1219-1227.

[12] S. Apprich, S. Trattnig, G.H. Welsch, I.M. Noebauer-Huhmann, M. Sokolowski, C. Hirschfeld, D. Stelzeneder and S. Domayer, Assessment of articular cartilage repair tissue after matrix-associated autologous chondrocyte transplantation or the microfracture technique in the ankle joint using diffusion-weighted imaging at 3 Tesla, Osteoarthritis Cartilage 20(7) (2012), 703-711.

[13] L. Filidoro, O. Dietrich, J. Weber, E. Rauch, T. Oerther, M. Wick, M.F. Reiser and C. Glaser, High-resolution diffusion tensor imaging of human patellar cartilage: feasibility and preliminary findings, Magn. Reson. Med. 53(5) (2005), 993-998.

[14] J.G. Raya, A.P. Arnoldi, D.L. Weber, L. Filidoro, O. Dietrich, S. Adam-Neumair, E. Mützel, G. Melkus, R. Putz, M.F. Reiser, P.M. Jakob and C. Glaser, Ultra-high field diffusion tensor imaging of articular cartilage correlated with histology and scanning electron microscopy, MAGMA 24(4) (2011), 247-258.

[15] R. Meder, S.K. de Visser, J.C. Bowden, T. Bostrom and J.M. Pope, Diffusion tensor imaging of articular cartilage as a measure of tissue microstructure, Osteoarthritis Cartilage 14(9) (2006), 875-881.

[16] K.J. Stevens, R.F. Busse, E. Han, A.C. Brau, P.J. Beatty, C.F. Beaulieu and G.E. Gold, Ankle: isotropic MR imaging with 3D-FSE-cube-initial experience in healthy volunteers, Radiology 249(3) (2008), 1026-1033.

[17] Z.V. Maizlin, J.J. Clement, W.B. Patola, D.M. Fenton, J.H. Gillies, P.M. Vos and J.A. Jacobson, T2 mapping of articular cartilage of glenohumeral joint with routine MRI correlation - initial experience, HSS J. 5(1) (2009), 61-66.

[18] Medical M. MITK 3M3 [Internet], 2009, available at: http://mint-medical.de/productssolutions/mitk3m3/mitk3m3.

[19] P.A. Cook, Y. Bai, S. Nedjati-Gilani, K.K. Seunarine, M.G. Hall, G.J. Parker and D.C. Alexander, Camino: open-source diffusion-MRI reconstruction and processing, in: 14th Scientific Meeting of the International Society for Magnetic Resonance in Medicine, Seattle, WA, USA, 2006, p. 2759. 
[20] A. Barmpoutis, B.C. Vemuri, T.M. Shepherd and J.R. Forder, Tensor splines for interpolation and approximation of DT-MRI with applications to segmentation of isolated rat hippocampi, IEEE Trans. Med. Imaging 26(11) (2007), 1537-1546.

[21] T. Shiomi, T. Nishii, H. Tanaka, Y. Yamazaki, K. Murase, A. Myoui, H. Yoshikawa and N. Sugano, Loading and knee alignment have significant influence on cartilage MRI T2 in porcine knee joints, Osteoarthritis Cartilage 18(7) (2010), 902-908. 